\title{
LA REVISTA DE HISTÓRIA ET QUELQUES PRISES DE VUE SUR L'HISTOIRE RÉCENTE - OU ANCIENNE? - DU BRÉSIL.
}

\author{
E. COORNAERT \\ du Collège de France; de la Académie des Inscriptions \\ et Belle Lettres. Ancien Professeur à l'Université \\ de São Paulo.
}

Faut-il vraiment un effort pour rassembler des souvenirs vieux de quarante ans? - Et si, amis paulistes, ils surgissaient de mieux que de la simple mémoire, mieux, oui, d'une source plus chaleureuse? Le seul difficile a été de trier dans un dense trésor. Un danger, je le sais: répéter des témoignages qui sont, aux regards des Brésiliens d'aujourd' hui, des vieilleries. Mais ils durent, en vérité toujours jeunes...

La Revista de História a 25 ans. L'âge de la maturité. Elle a grandi vigoureuse, sage dans un monde du vertige. Depuis 1950, elle nous apporte chaque trimestre des fruits de bonne et belle forme. La première d'Amérique latine, à un rang des plus honorable parmi tous les périodiques mondiaux d'histoire.

Les semailles avaient eu lieu quinze ans plutôt. São Paulo disposait depuis longtemps d'une faculté de droit, chaque jour nécessaire; l'Etat venait de pourvoir sa faculté de médecine de beaux immeubles modernes qui allaient héberger d'abord une nouvelle venue, la faculté de sciences et lettres. Le projet de celle-ci se pesait depuis bien des années. En 1932, les libéraux de l'époque dirigeaient l'Etat: le partido constitucionalista souleva le pays contre une "dictature" (c'était l'époque de ce genre d'“ordres"). L'événement n'eut-il aucune part au fléchissement décisif de la balance en faveur du dessein encore en l'air?

La nouvelle faculté comprit au départ sept Français pour les lettres, pour les sciences trois Allemands et un Italien, tous quatre heureux d'échapper à leurs régimes. Des Français, le seul à connaître le pays avait été en 1933 à Rio la coqueluche des milieux cultivés par une série de brillantes conférences; les six autres faisaient leur "découverte de l'Amérique". 
Nous arrivions par mer: 1934. A y penser, je suis encore saisi par la longue et envoûtante traversée le matin tôt, dans une lumière légèrement tamisée, du chenal de Santos par l'exubérante végétation, pour nous exotique, de palmiers, palétuviers, bananiers, etc. etc., par l'entrée dans l'océan vert aux mille nuances qui s'étend sur l'immense Brésil.

São Paulo, la cidade, était encore en train d'inaugurer sa révolution urbaine sans égale, plus rapide que celle de New-York. Il subsistait encore quelques rues aux noms évocateurs de fonctionnaires de jadis, rues de modestes maisons basses sans étage: on pouvait y rêver des "temps coloniaux", y revivre ce lointain pourtant proche, qui s'enfonçait dans la nuit du passé et le faisait sentir encore. Les ypês déroulaient dans les avenues leurs guirlandes enchanteresses. Avec ses vingt-deux niveaux, le Martinelli faisait figure de géant; une quinzaine d'autres tours s'égaillaient de ci de là. D'après une récente photo aérienne, le géant de naguère fait figure de petit garçon "demeuré" dans la compacte forêt de colosses qui fait de São Paulo un "ensemble" unique sans dessin précis, "qui s'est fait et défait dix fois depuis un siècle", où autostrades, ponts et enjambements s'entrecroisent de toutes parts, doté d'ailleurs par ses architectes (qui ont cessé depuis longtemps de venir s'instruire à Paris) de prestigieuses constructions inspirées d'heureuses traditions nationales: "la locomotive...".

Nous étions là pour enseigner. Elle n'a pas baissé d'un cran depuis 1934, mon admiration pour l'assiduité des 35-40 étudiants habituels. Leur évidente ardeur, leur appétit d'apprendre jouaient sur le maître comme un actif tonique ... Je vois encore en haut des travées près de la fenêtre, prenant des notes avec conscience - décidé à ne citer aucun nom propre, je m'arrête devant le sien - celui qui est depuis 25 ans la force et l'âme de cette revue. (Devant la chaire, en bas, un autre suivait avec la même attention, mais décidé, lui, à bifurquer vers une autre discipline). Mon programme portait sur le Moyen Age et les Temps Modernes, vaste "territoire", comme on dit au;ourd'hui. Ce que je me rappelle le mieux est de leur avoir "appris" Henri Pirenne. Le célèbre historien était alors à un zénith mérité par l'ampleur et la chaleur de ses vues. (Au vrai, malgré mon admiration, qui n'a pas décliné, malgré mon affection toujours fidèle, j’avais commencé, lun des tout premiers, je crois bien, à porter la main sur l'arche sainte, quelques somptueuses synthèses: draperie flamande, corporations et liberté, capitalisme du XVIe siècle et Réforme, synthèses qui ont subi le sort de tous les vastes panoramas d'histoire, féconds, sauf rares exceptions, la durée d'une génération).

Un souvenir particulier me fait revivre l'examen de fin d'année. De ces étudiants de choix, il en était qui, comprenant fort bien le fran- 
çais, étaient timides pour le parler. Il fut convenu qu'on pourrait me répondre en portugais - pardon! en brésilien. Pour plus de sûreté, un "assistant", éventuel interprète, rassura les interlocuteurs: il n'eut pas à intervenir.

Si j'ai enseigné, j'ai aussi beaucoup appris dans ce pays si neuf. Tout d'abord, bien sûr, le Padre Anchieta, l'extraordinaire épopée des Bandeirantes, l'exceptionnel Alejadinho (Congonhas, Ouro Preto, Sabará... ), Tiradentes, dom Pedro, l'enveloppant $O$ Sertão d'Euclides da Cunha, qui laisse après lui tant de saudades avec l'impression d'un monde sans fin (impression ranimée par le Retrato do Brazil de Paulo Prado: percutant dès la première phrase, $O$ povo brazileiro é um povo triste... ce qui n'est vrai qu'en partie).

Le plus sûr, le plus pénétrant enseignement acquis là-bas peut, je crois, étonner d'abord. Nulle part je n'ai senti comme chez ce futuriste déchaîné, le Brésil, ce que fut chez nous le Moyen Age: la fazenda, la maison du maître (le terreiro, il est vrai, brouillait un peu les analogies, mais à peine), à côté, l'église presque toujours construite par lui et lui procurant d'ordinaire au moins un droit de regard sur la nomination du curé, les "colonies" - futurs villages chez nous dispersées dans toute l'étendue du domaine (le plus étendu que nous ayons visité, grand comme tout le département de la Seine d'alors (aujourd'hui cinq) et le samedi après-midi les femmes des colons allant au centre faire dans les vendas les provisions de la semaine le long des pistes, les seules routes (Ah! Ces pistes et ces routes, résumés de l'histoire du pays! J'entends encore le prefeito de Rio Preto, déjà ville importante, me disant en voiture avec une pointe d'envie compréhensible: "A l'est, ils ont des estradas bem calçadas". Depuis lors...!).

Le Moyen Age, nous l'avons vécu en groupe intensément à São Paulo par une excursion aux confins extrêmes - alors - de la civilisation, - une excursion comme les Brésiliens en ont sans guère de doute entendu raconter à foison ... Un de nos amis, parmi d'autres d'une distinction, d'une finesse d'esprit et de coeur exceptionnels (décédé voici quelques années fazendeiro éleveur de buffles) était en excellents termes avec une compagnie anglaise qui défrichait dans l'ouest de l'Etat. Il obtint pour notre équipe un petit voyage de trois jours à Londrina, alors en train de naître.

Embarquement le soir, arrivée le matin à Jatahy, ancien poste militaire, ni ville ni village, perdue dans une région à peu près vide. Deux ou trois cheminots à la gare, personne dans les rues, - si, un Français d'un certain âge, sans guère de doute d'un statut légal moins certain, - dans un coin d'une place au contour écorné de plusieurs 
vides un engin étique, un pauvre manège de chevaux de bois fermé,... dans cette quasi solitude. Le train de la compagnie nous attendait: un beau tacot encore presque neuf, pour voie étroite. Deux d'entre nous s'installèrent, jambes ballantes, sur le devant de la locomotive; une bonne quarantaine de kilomètres sans une gare, sans une halte, sans l'ombre d'une vie humaine apparente dans la forêt, la forêt, la forêt, deux murailles vertes bordant le mince ruban de voie, une longue saignée au coeur de la forêt-vierge. Passionnant. Tout d'un coup, Londrina: une vaste clairière, des souches émergeant de tous les côtés; maisons en bois, église en bois, une seule maison en dur; notre hôtel, en bois, assez confortable; les chambres, des cabines de navire.

Et là, des histoires de la région (?) à n'en pas finir: des Indiens qui apparaissaient subitement entre les arbres, disparaissaient, insaisissables; à bonne distance "en avant", un caboclo vivant avec une femme dans des conditions extravagantes en dehors de toute société. $\mathrm{Au}$ delà, nous assura-t-on, plusieurs jours de marche dans le fouillis serré, inextricable ou tout comme, avant le véritable sertão. "Vraiment"? ... Aujourd'hui Londrina est, paraitt-il, une belle ville pourvue de son aéroport. Et bien plus loin, ayant passé par le même stade, Marília est, elle aussi, une belle ville qui a sa faculté des lettres et sa revue d'histoire, fille de "la nôtre", fondées par qui nous savons.

Voici quarante ans, un Européen au Brésil sentait presque physiquement que l'homme commandait encore difficilement à la nature ... En ce temps-là, Brasília était à peine une idée du très petit nombre et l'Amazonie restait, encore à peine sondée, un monde du mystère.

L'histoire "s'accélère" partout, - au Brésil depuis un demi-siècle plus vite qu'ailleurs...?

Le Moyen Age du Brésil, je l'ai "expérimenté" à Paris trois ou quatre ans après l'avoir entrevu à São Paulo. Une étudiante de "notre" faculté pauliste vint à Paris préparer un "diplôme" de l'Ecole pratique des Hautes Etudes. A la IVe section alors, on ne "descendait" pas en deçà de 1789 . Je proposai à l'assemblée le sujet du travail: le titre parlait de 1860. Sentencieusement, le président objecta, au vrai, moins sûr de lui qu'à l'accoutumeé: " 1860 ? Ce ne sont pas des sujets dont nous nous occupons ici". Je dis simplement: "Mais, 1860, là-bas c'est bien le Moyen Age". Un bon éclat de rire fit admettre le sujet, puis passer le travail, qui était fort bon. 


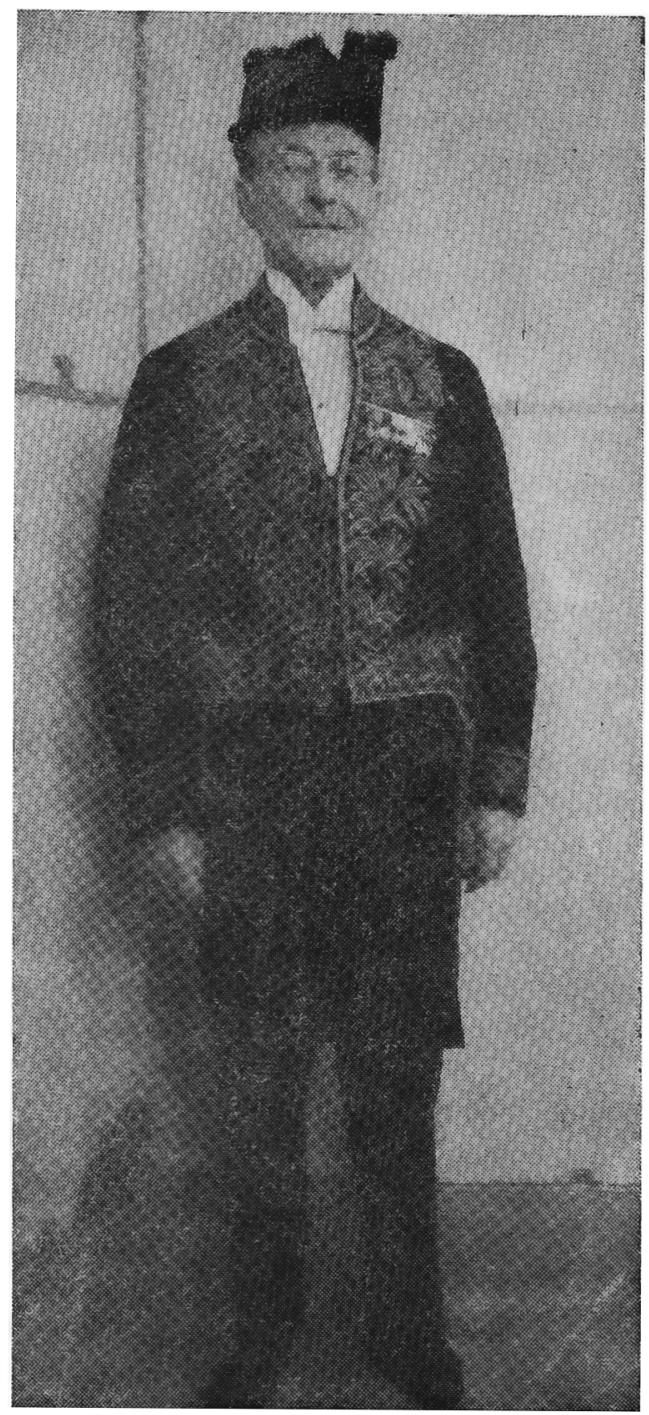

Emile Coornaert, o 19 Professor de História da Faculdade de Filosofia, Ciências e Letras da Universidade de São Paulo, concorreu e muito para imprimir-lhe as diretrizes que a distingue no contexto cultural brasileiro. Havendo atuado efetivamente em 1934 e 1949 e afetivamente até nossos dias, pois de Paris, onde reside, vem mantendo comovente correspondência com seus ex-alunos, hoje seus sucessores. 
Ao homenagear o pioneiro dos mestres estrangeiros na imposibilidade de se captar instantâneos de muitos outros, escolheu-se o retrato que o clichê focaliza. Ei-lo, com a farda da Academia de Ciências e Letras, onde se pode perceber 9 medalhas. Gloriosamente expressivas. Combatente de duas Grandes Guerras. Ferido. Impôs respeito, admiração aos próprios adversários. Filho de uma região fronteiriça, com invulgar capacidade linguística, chegava a interceptar mensagens dos alemães. Provado e comprovado que é um homem de ação. Combativo. Todavia ao se observar o seu olhar de frente, que as lentes bifocais acentuam, pode-se captar a mensagem daqueles que, por força do seu próprio exemplo, sobrevivem e ajudam outros sobreviver nas horas de crise que ameaçam até as ciências humanas. (Nota de Maria Regina).

\section{EMILE COORNAERT. Né à Hondschoote (Nord) le} 31.8.86.

Etudes: St François d'Assise (Hazebrouck), Facultés Catholiques de Lille, Sorbonne.

Grades: Agrégé d'histoire, docteur ès Lettres.

Postes: Notre-Dame des Dunes à Dunkerque, Collège Stanislas (Paris), Lycées Alençon, Nancy, Condorcet (Paris), directeur d'études à l'Ecole Pratique des Hautes Etudes, professeur au Collège de France, membre de l'Académie des Inscriptions et Belles-Lettres.

Ouvrages: Réédition de La Dixme royale de Vauban; La draperie-sayetterie d'Hondschoote; Les corporations en France jusqu'en 1791; Les hommes au travail; Les Français et le commerce international à Anvers (XVe-XVIe siècles), 2 vol.; Les compagnonnages; La Flandre francaise de langue flamande, articles et comptes rendus dans revues diverses.

Missions: Brésil, Belgique, Pays-Bas, Angleterre, Allemagne, Suisse, Italie, Pologne, Canada, Argentine, Chili.

Distinctions: Officier de la Légion d'Honneur, Commandeur de l'Ordre National du Mérite, Croix de Guerre 14-18, médaille de la Résistance, diverses autres françaises, Commandeur de Polonia restituta, chevalier de l'Ordre de Léopold. 